\title{
Study on Multi-condition Test of Wheel-rail Adhesion Coefficient Testing Machine
}

\author{
Zha Dong \\ Practice Training Center \\ East China Jiaotong University \\ Nanchang, Jiangxi,China \\ E-mail: 710913621@qq.com
}

\begin{abstract}
This paper presents a scheme of multi-conditions of wheel-rail adhesion ball-disc testing machine. It is composed of mechanical components, hydraulic components, control components and data processing system, and can be carried out different testing experiments under the conditions of different speed, temperature, humidity, loading, magnetic field, materials, lubricant and slide roll ratio. And it also can complete the rail material adhesion test conditions under the coupling action, in the experimental process, in the course of the experiment only through the different conditions combination, the adhesion coefficient can be tested under the coupling action of different conditions; At the same time using the data processing system, the test data are analyzed in the process of testing, realizing real-time graphics output and data storage and other functions.
\end{abstract}

Keywords-Multi-conditions; Wheel/rail interaction; Adhesion coefficient; Ball/disk friction

\section{INTRODUCTION}

In the recent few years, with the gradual increase of train speed, more and more domestic and foreign scholars begin to deeply study the problems of high speed wheelrail adhesion. The adhesive force of wheel-rail could directly affected the stability, stationarity and safety of train operation, which is a must factor for realizing the train traction, operation and braking, thus it is extremely important to acquire the wheel-rail adhesion coefficient precisely. Back in 1980, Pritchard published a world wide research summarize of wheel-rail adhesion problems in the seventh Leeds-Lyon Tribology seminar, discussed the influences of wheel-rail adhesion from multi-factors. Later, many of Japan scholars adopted the test method to study the affections of wheel-rail adhesion from wheel-rail parameter, rolling speed, dry and lubricate conditions and other pollutants between wheel and rail. Nagase $\mathrm{K}$ designed a special bogie and installed on the train to do wheel-rail contact surface tests in multi-conditions, finally he found that in rainy day, the adhesion coefficient would decrease sharply, and the high the rolling speed is, the smaller the adhesion coefficient is. Akira Matsumoto used small scale wheel-rail test-bed to study and found that the maximum friction coefficient is 0.46 in clean contact condition, and it is 0.16 in abrasives condition. Koan-Sok Baek imitate the wheel-rail contact through double disk of roll unit, the change of the traction coefficient during the process of imitation test are mainly caused by the third medium like surface roughness, surface hardness, the oxide layer of contact surface and so on. Chen $\mathrm{H}$ and some other people chose real steel rail material to imitate the actual working conditions with the same parameter through test machine. From the test he found that under water polluted condition, the wheel-rail surface roughness and water temperature increase, wheel-rail adhesion presented a rising tendency. In the following study, they found something new that with the increase of trial speed, the wheel-rail coefficient would drop. Except the Japan scholars did the research and work above, scholars from other countries including China also did a huge amount of test and research for the wheel-rail adhesion problems. Qian Lixin, who is from China Academy of railway sciences, presented the imitation technology of wheel-rail in laboratory during his further study in America inn 1981, he found that with the magnify of the angle of attack, the lengthways adhesion coefficient decrease obviously through curve zone, the adhesion coefficient would decrease greatly when there is oil and water pollution on the wheel-rail surface. Zhang Weihua and some other people, who are from SWJTU, utilized imitation test machine to analyze how the parameter of Axle load, wheel diameter, viscous block and the angle of attack of wheelrail of locomotive affect the wheel-rail adhesion and creep characteristic, he found that once increase the axle load, decrease the wheel diameter, increase the angle of attack of wheel-rail, the wheel-rail adhesion coefficient would drop. Jin Xuesong and some other people used the high speed wheel-rail adhesion test system to do a adhesion mechanism research under the surface of wheel-rail with water medium condition, they got the adhesion coefficient from different speed,and discussed about the gap between the test result and the calculate result. Recently,Zhang Hongfei and some teammates used JD-1 wheel-rail test machine to how the magnetic field in water and oil medium condition affect the characteristic of wheel-rail adhesion. Gallardo-Hernandez and his teammates, who are from England, adopted the double disk test machine to discuss about affection of wheel-rail adhesion from water, oil, moist leaf and sand, but no one do a specific research aiming at the influence brought by environment temperature and humidity.

From the domestic and foreign study situation, the study of wheel-rail is always by means of test, and we are by means of equal or small scale large wheel-rail friction test-bed for the wheel-rail adhesion multiple loading condition imitation, but the cost of making and testing of large wheel-rail friction test-bed is incredibly expensive, the ordinary research and development institution can't not 
afford it, and the almighty friction test machine that we have currently could not realize wheel-rail multiple loading condition imitation. Therefore, this passage base on the ball-on-disk wear multiple loading condition wheelrail adhesion coefficient test machine, which could measure the ball-disk adhesion coefficient in different loading conditions like speed, temperature, humility, load and lubrication. This test machine could extend the balldisk usage life through special device adjust the ball-disk contact position.

\section{THE WORKING PRINCIPLE}

Adhesive coefficient is the static friction coefficient on the locomotive traction wheel and rail contact, the sliding friction coefficient of tending to zero, namely relative velocity in the study of some adhesive coefficient can be substitute coefficient of friction. This article will of steel ball rolling on the disk is equal to the adhesive coefficient of friction coefficient, defined as the contact point of the tangential force and normal force ratio;

$$
\text { Namely: } \mu_{\text {剩着 }}=F_{T} / F_{N}
$$

$\mu_{\text {黏着 }}$ is adhesive coefficient to them, $F_{T}$ is the tangential force, $F_{N}$ is the ball at contact point by the normal force of [4].

Experiments, through the force of the force transducer will contact real-time transmission to the data processing system, and real-time display of adhesive coefficient values.

Testing machine can realize the working condition of several main have different speed, different humidity, temperature, load, different lubricants, roller ratio, different materials and different magnetic fields. The principle respectively under various working conditions is as follows:

Different speed and different roller than: implement different speed and different working condition of roller than the main is to control the speed of motor, drive disc motor and drive the ball to give different current, in different speed on the disk drive the ball rolling.

Different humidity: the conditions of different humidity mainly inside the ball plate work parts of the body, additional humidity controller, when need to change the housing internal humidity, injection water mist, the humidity controller with humidity sensor, when the humidity reaches a certain degree of stop injection [4,5].

Different temperature: the work environment to increase in ball set heating, cooling, and temperature feedback and control device in order to realize the regulation of different temperature [3].

Different load, different load cases are mainly control the hydraulic system of the motor rotation speed and different rotational speed of the motor to make the amount of oil pump in the pump body to the hydraulic cylinder, hydraulic cylinder on the top of the piston upward force, so as to realize the simulation of different load [6].

Different lubricants: through additional lubricant on the upper box body add device and ultrasonic cleaning device, a kind of lubricant being released from a hole at the bottom of the box body after use, in order to avoid the influence on a kind of lubricant, lubricant residues on the switch different lubricants using ultrasonic device to a kind of lubricant thoroughly clean thoroughly before adding additional lubricant [7].

Different materials: the working principle of the is by changing the steel balls of different materials and different materials of the disc to achieve [8].

Different magnetic field: the working principle is placed inside the box body coil, the influence of different magnetic field on the adhesive coefficient needs to be done, the current coil zhongtong into different sizes to achieve the working condition of [10].

\section{CONSTITUTION OF THE TRIAL MACHINE SYSTEM CONSTITUTION}

The machine consists of mechanical parts, hydraulic parts, ball plate work device, control part and data processing system, figure 1 for testing machine overall structure diagram.

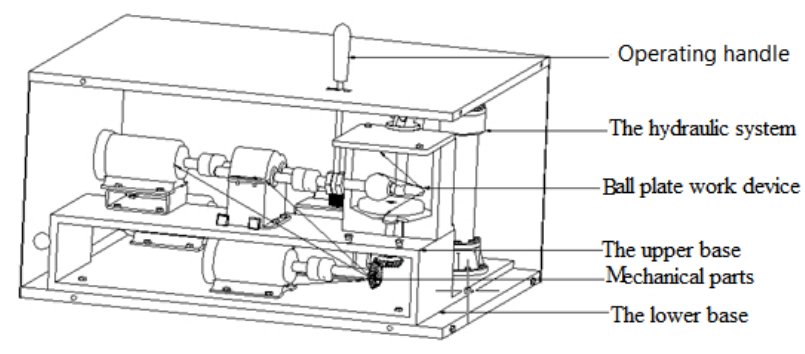

Figure 1. Machine overall structure diagram

Mechanical part of the figure 1 shows that the two motor respectively fixed at the side of the base of the lower level, form two independent drive system; The other side of the hydraulic part on the base, and the hydraulic system of all the specimens were fixed in the same horizontal plane; Ball device fixed on the base of the upper plate work, through the load imposed rod with hydraulic parts.

\section{KEY STRUCTURE OF THE TRIAL MACHINE}

The key structure of trial machine contains mechanical components, hydraulic components and ball disc working device.

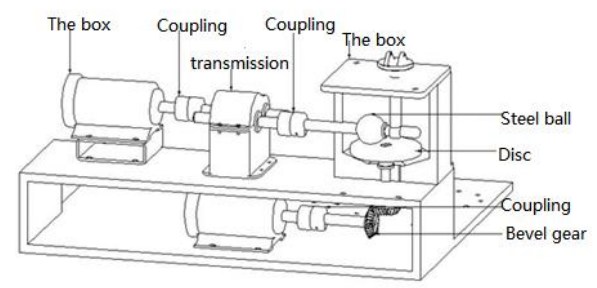

Figure 2. The mechanical parts of the machine structure diagram

The function of mechanical components is to realize the accurate work of ball/disc device, through the motor, the coupling and the bevel gear pair etc. to achieve the rotation of the disc. Also through the motor, reducer and coupling, etc. to achieve the ball rolling on the disc. The driving device for connecting the steel ball and the box body are fixed on the base plate; the driving device for connecting the disc is fixed on the base layer. 


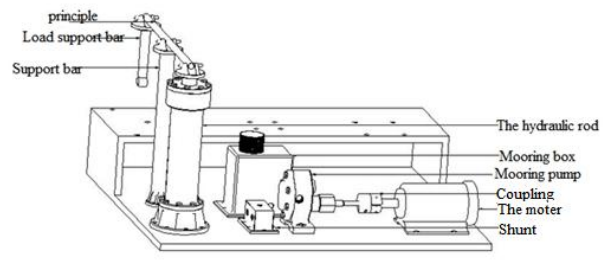

Figure 3. Machine hydraulic parts structure diagram

The main function of hydraulic components is to apply load. The hydraulic pump start-up driven by motor after it being powered, the piston rod in hydraulic cylinder moves upward, as shown in Figure 3, taking advantage of lever principle, while the load is applied, the other end of the bar will press down. Then the load is applied to the contact zone of the disc and steel ball, thus, can show the influence of adhesion coefficient by different load.

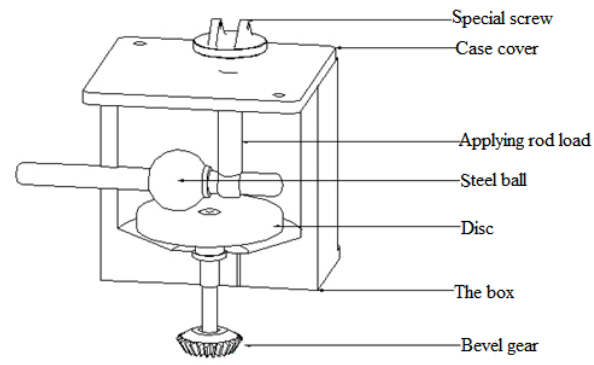

Figure 4. Tester structure schematic experimental section

Ball/disc working device of trial machine, mainly include the box, steel ball and disc. In this part, the movement of the steel ball and disc is the foundation to test the adhesion coefficient under different conditions. The key technique of this part is the replacement of steel ball and disc, the special screw and the load exerting bar are connected by bolts as shown in Figure 4,unscrew the special screws before opening the box, take the load exerting bar off after opening the box. The ball and the bar which in the rotation of it are fixed by a pin, remove the pin you can take the steel ball off, and then take the screws out in the centre of the disc, you can take the disc off.

In order to reduce the trouble of replacing the steel ball and the disc, the trial machine use the wheel/ rail ball/disc mechanism to change the contact position, as shown in Figure 5,design a operating handle to change the position, the operating handle located in the contact place of the box body at the beginning. After one test is finished, the experimental circle on the disc can not be used again. By handling the operating handle, take a movement to the left, the rubber gasket under the ball moving bar can be fixed again through the clamping groove outside of the box, the ball can carry out a new circular motion on the disc. The contact part between the ball moving bar and the ball connecting bar is a lantern ring, which does not affect the ball's rolling. The position of the ball can be moved through the two fixed nut, the axle connected with the operating handle is connected with an axle of the transmission through a coupling. The gear width on the axle is smaller, while the other gear width is larger, to prevent the contact problem of the gear after moving the ball.

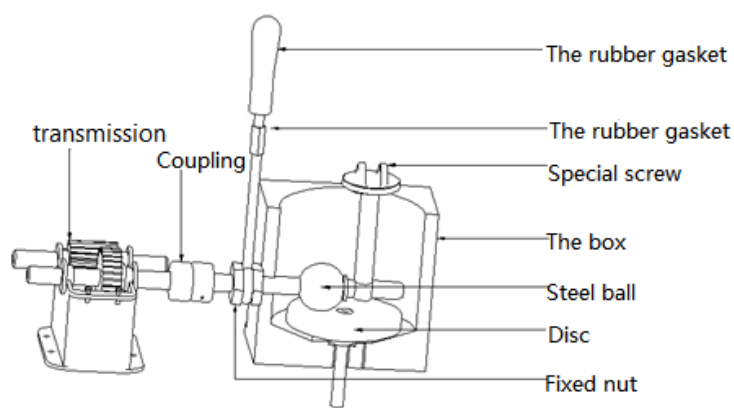

Figure 5. Wheel/rail ball map sample contact position exchange mechanism

\section{CONCLUSION}

More working condition of wheel/rail adhesive coefficient of the most important function of testing machine can be very convenient to realize simulation of wheel/rail adhesive more conditions. In this paper, the wheel/rail adhesive machine adopts the ball disc wheel/rail friction method and mechanical and electrical technology can implement more convenient and fast operating mode under the condition of wheel/rail test stick together, and innovative are sample contact position exchange mechanism of wheel/rail ball prolonging the service life of ball tray, which can effectively reduce the wheel/rail friction test cost.

\section{REFERENCE}

[1] PRITCHARD c. Traction Between Rolling Steel Surfaces - A Survey of Railway and Laboratory Services, F, 1980 [C]. 7th Leeds - Lyon Symposium an Tribology, Leeds, 1980.197 206.

[2] Xue-song Jin, Qi-yue Liu. Tribology of wheel and rail [M]. Beijing: China railway publishing house, 2004.A,

[3] MATSumOto SATO Y, ONO H, et al. The Creep Force Characteristics between Rail and Wheel on Scaled Model [J]. Journal of Wear, 2002, 253 (1-2) : 199-203.

[4] BAEK K S, NAKAHARA t. KYOGOKU K, An experimental study of transient traction characteristics in rolling - sliding wheel/rail contacts under dry, wet the conditions [J]. Journal of Wear, 2007, 263 (1-6) : 169-179.

[5] CHEN H, the number M, NAKAHARA t. Analysis of adhesion under wet the conditions for three - dimensional contact considering surface roughness [J]. Journal of Wear, 2005, 258 (78) : 1209-1216.NAMURA

[6] CHEN H, the number of M, A, et al. The Estimation of wheel/rail adhesion coefficient under wet condition with measured boundary friction coefficient and real contact area [J]. Journal of Wear, 2011, 271 (1) : 32-39.

[7] KUMAR S, Li-xin Qian. Laboratory Simulation of Wheel/rail Contact Parameters and the Wheel/rail Impact Angle, sinusoidal movement, oil and water pollution, the real contact area on the properties of adhesion, creep effect $[\mathrm{J}]$. China Railway Science, 1984, 5 (1) : 12-39.

[8] Zhang Weihua, Drucker. The Mechanism of Wheel/Rail Adhesion Test Research [J]. Journal of railway, 2000, 22 (2) : 20 to 25.

[9] Xue-song Jin. Wheel-rail creep theory and experimental study [M]. Southwest Jiaotong University press, 2006.

[10] XIAOYU J, XUESONG. for Numerical simulation of wheel rolling over rail at high speeds - [J] Journal of Wear, 2007, $262(5-$ 6) : 666-671. 